\title{
High performance Brillouin distributed fibre sensor
}

\author{
S. Diaz*b ${ }^{\mathrm{b}}$, S. Foaleng Mafang ${ }^{\mathrm{a}}$, M. Lopez-Amo ${ }^{\mathrm{b}}$, L. Thévenaz ${ }^{\mathrm{a}}$ \\ ${ }^{a}$ Nanophotonics and Metrology Laboratory, École Polytechnique Fédérale de Lausanne \\ STI-NAM Station 11, CH-1015, Lausanne, Switzerland \\ ${ }^{\mathrm{b}}$ Dep. of IEE, Public University of Navarra, Campus de Arrosadia s/n, 31006 Pamplona, Spain
}

\begin{abstract}
We propose a novel configuration for a Brillouin distributed sensor based on Brillouin optical time domain analysis. This new configuration eliminates many intensity noise issues found in previous schemes. Resolution of $3.5 \mathrm{~m}$ all over a $47 \mathrm{~km}$ single-mode fibre was achieved and resolution down to $30 \mathrm{~cm}$ in a few kilometre fibre. Noise reduction makes possible measurements with a 16 times averaging.
\end{abstract}

Keywords: Fibre optics, stimulated Brillouin scattering, fibre sensors, nonlinear optics.

\section{INTRODUCTION}

Fibre distributed sensors are gaining a lot of interests for applications in various fields, such as structure monitoring, pipeline integrity check and geotechnical applications to secure ground stability. This stimulates the development of configuration offering better performance in terms of range, acquisition time and spatial resolution, while keeping the cost of the instrument and the operation simplicity unchanged.

A Brillouin Optical-fibre Time-Domain Analyser, or BOTDA, was initially developed as an instrument enabling nondestructive evaluation of optical fibre attenuation ${ }^{1}$, based on the Brillouin interaction between two counter-propagating lightwaves generated by two laser sources placed at the extremities of the fibre under test.

The majority of methods for BOTDA ${ }^{2,3}$ has used two distinct lasers for generating pump and probe signals. This requires an excellent frequency locking between the two lasers to secure the $1 \mathrm{MHz}$ stability for accurate measurement. Niklès, Thévenaz and Robert ${ }^{4,5,6}$ proposed a simple way to achieve an ideal stabilization of the frequency difference in a BOTDA. The configuration uses a microwave generator and a $\mathrm{LiNbO}_{3}$ electro-optic modulator (EOM) to generate pump and probe signals from one single laser source. The EOM modulated the laser light at frequency near the Brillouin frequency shift, to generate a probe wave from the modulation sideband. The same EOM also produced a pump pulse by applying an electrical pulse to the EOM electrodes. The configuration is very compact, shows an inherent excellent stability and requires very few optical elements. The pump and probe lightwaves propagate back and forth within the sensing fibre taking advantage of a passive reflection at the fibre far end. This made single fibre end measurement possible.

This configuration however suffers from sensitivity to optical noise, as a result from the bidirectional propagation of optical waves showing the same frequency along the optical fibre. A fraction of any of these optical wave may propagate in the opposite direction through partial spurious reflections at splices and connections or simply through Rayleigh backscattering. These backreflected contributions superpose to the optical waves normally propagating in the reverse direction. The superposition with a wave showing the same optical frequency gives rise to interferences that turn into intensity noise since the fibre is usually much longer then the laser coherence length. The most likely situation is thus that the phase difference between the superposed is totally random, resulting in a rapidly changing chaotic interference. Considering the small amplifications observed through Brillouin gain - in the percent range - the intensity noise on the detector may easily be comparable in amplitude with the gain contrast.

This feature actually limits the performance of the system in terms of range, spatial resolution and measurement time. It was decided to investigate a novel configuration that avoids as much as possible the bidirectional propagation of waves showing the same optical frequency

Third European Workshop on Optical Fibre Sensors, Antonello Cutolo, Brian Culshaw, José Miguel López-Higuera, Eds., Proceedings of SPIE Vol. 6619, 661938, (2007) $\cdot 0277-786$ X/07/\$18 $\cdot$ doi: 10.1117/12.738661 


\section{EXPERIMENTAL SETUP}

The main original feature of the setup is the presence of a single laser source that is separated into 2 fibre channels: one is modulated through an electro-optic modulator (EOM) to generate the $\mathrm{CW}$ probe lightwave, the other is gated by a SOA to make the pump pulse. This gives to the system an inherent stability, as far as frequency drifts of the laser are concerned. The pump pulse provides gain to the probe signal during its forward propagation through the stimulated Brillouin scattering process while the $\mathrm{CW}$ probe is amplified on the way back.

The block diagram of the setup is shown in Fig. 1. The laser light is first split into probe and pump channels. The probe light is modulated by an electro-optic modulator (EOM). This is a key element of the setup since it is used for the generation and frequency tuning of the probe signal. The frequency shift on the laser light is achieved by simply applying a CW microwave signal in the frequency range of the Brillouin shift on the electro-optic modulator electrodes. This creates sidebands in the laser spectrum. This technique makes the control of the probe optical frequency very convenient and reliable by adjusting the microwave modulation signal frequency. The DC bias setting on the electrooptic modulator just determines the amount of transmitted amplitude at the fundamental frequency. The DC bias condition on the EOM electrodes is set, so that the optical power at the laser original wavelength is kept as small as possible. This minimal power amount depends on the EOM characteristic extinction ratio that is specially selected to exceed $35 \mathrm{~dB}$.

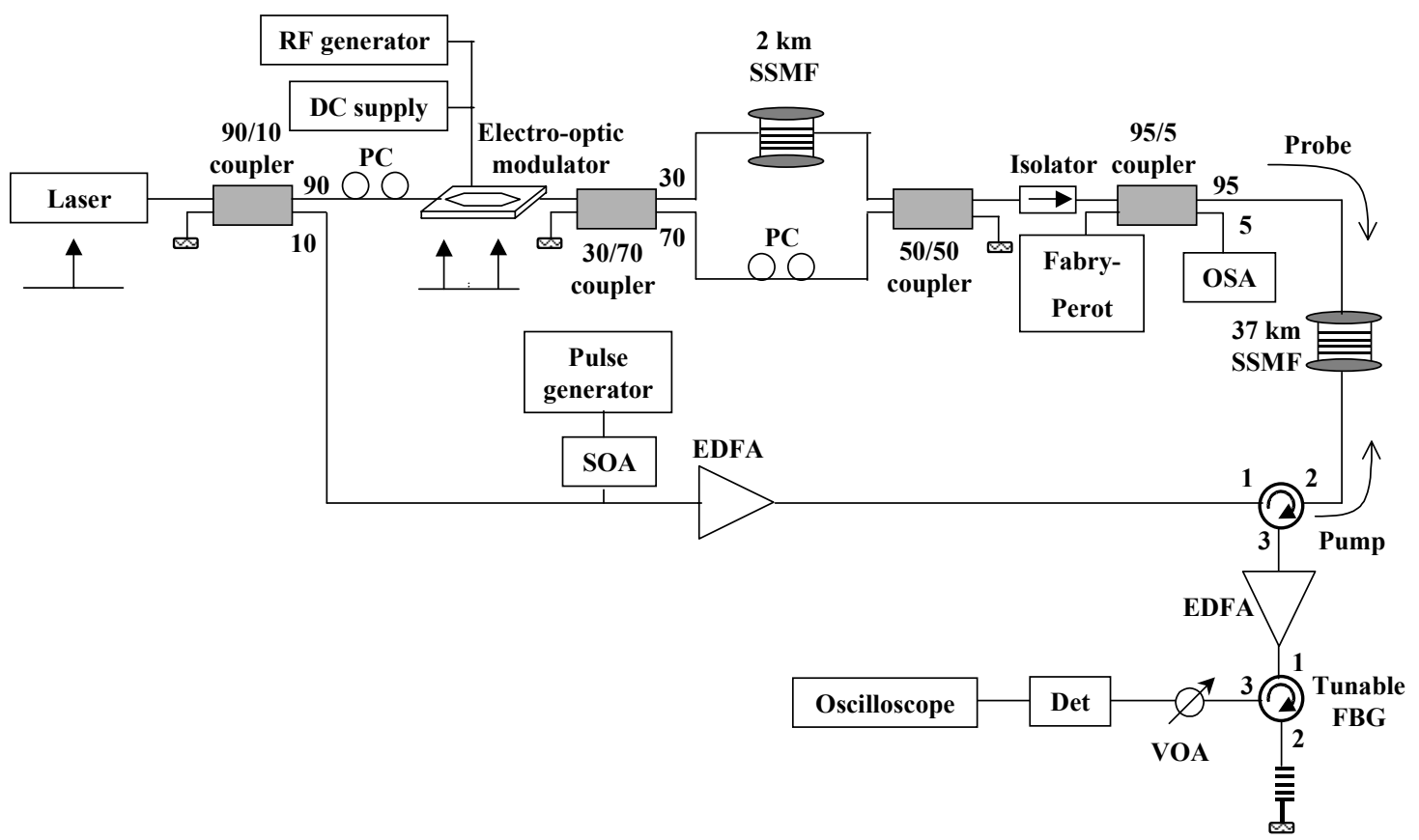

Fig. 1. Novel low-noise configuration for distributed Brillouin gain spectrum measurement

To avoid any gain fading effect resulting from the polarization-dependent Brillouin interaction, we have built and developed a new passive polarization scrambler. It consisted in two branches, one with a $2 \mathrm{~km} \mathrm{SSMF}$ and another with a polarization controller, and two couplers, resulting in a highly unbalanced Mach-Zehnder interferometer. The arm length difference is much larger than the laser coherence length, so that they combine incoherently in the output coupler. The states of polarization in the 2 arms are set orthogonal before the recombination, so that the phase noise results in a perfect and passive polarization scrambling without intensity noise. It was placed between the EOM and the isolator, where the light is no longer subject to polarization dependent elements. It must be pointed out that this kind of passive scrambling is absolutely perfect, but is restricted to $\mathrm{CW}$ waves.

In the other channel the pump pulse is generated by a SOA, in which the gain is gated using a train of electric pulses. The SOA shows an ON-OFF ratio over $40 \mathrm{~dB}$, so that the presence of light at the original laser frequency is extremely low when the SOA is in OFF state, i.e. when the pump pulse is off. In addition there is absolutely no light generated at the 
sidebands frequencies. Hence the configuration secures a total absence of intensity noise at the sideband frequencies that were the main source of noise in the former configuration. The output is then boosted by an EDFA.

The signals from each channel are then directed to each end of the sensing fibre. There is conceptually no wave showing the same frequency propagating in the two opposite directions through the fibre, except the small residual light at the original laser frequency that results from the finite extinction ratio of the EOM, which can interfere with the Rayleigh light from the intense pump pulse.

Light from the probe channel is extracted at the fibre output using an optical circulator. This signal is levelled up using another EDFA and then filtered using a very narrowband fibre Bragg grating filter $(<0.1 \mathrm{~nm})$ to transmit only one sideband onto the detector. This filtering is crucial: it eliminates the unwanted modulator sideband that reduces the measurement contrast and any presence of the pump frequency due to the finite extinction ratio of the EOM that would generate substantial optical noise at the detection when combined with the Rayleigh light from the pump pulse.

When the modulation frequency $f_{m}$ is close to the Brillouin frequency shift $v_{\mathrm{B}}$, the first lower sideband lies in the Brillouin gain spectrum generated by the pump and is amplified through the Brillouin interaction. As a matter of fact, the Brillouin gain spectrum can be determined by simply sweeping the modulation frequency $f_{m}$, and recording the probe intensity. The frequency spacing between the first upper and first lower sidebands is twice the frequency modulation $f_{m}$, (approximately $22 \mathrm{GHz}$ ), and corresponds to a wavelength separation of $0.25 \mathrm{~nm}$ at $1.55 \mu \mathrm{m}$. As the first upper sideband is not relevant for the measurement and even has a negative effect on the contrast, it is filtered out just before acquisition using a tuneable Fibre Bragg Grating of $0.1 \mathrm{~nm}$ bandwidth.

\section{RESULTS}

The main performances of our Brillouin optical fibre system may be summarised as follows:

- The massive reduction of the noise down to optical shot noise has made a $50 \mathrm{~km}$ sensing range possible without any amplification along the sensing fibre to restore the pump power (see Fig.3).

- The ultimate spatial resolution of the Brillouin sensor depends on the fibre length and amounts roughly to $2 \mathrm{~m}$ for $40 \mathrm{~km}$-range and $3.5 \mathrm{~m}$ for $50 \mathrm{~km}$. For ranges shorter than $5 \mathrm{~km}$, a $30 \mathrm{~cm}$ resolution was obtained.

- For short ranges $(<5 \mathrm{~km})$ a $16 \mathrm{X}$ averaging results in perfect measurements, as shown in Fig.2. For very long ranges $256 \mathrm{X}$ averaging is required.

- A new passive polarization scrambling method was successfully implemented, resulting in a perfectly flat amplification profile along the fibre and reducing polarization fading to zero.
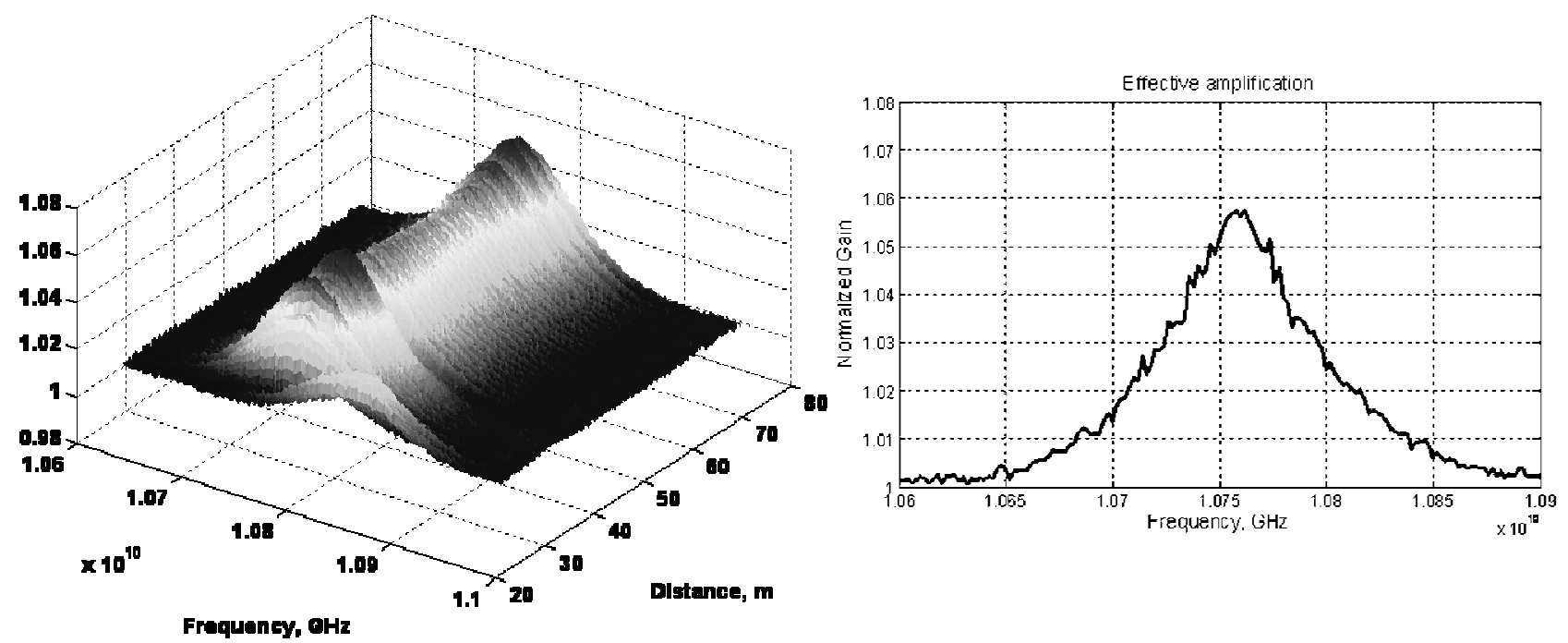

Fig. 2. Left: $3 \mathrm{D}$ view of the distribution of gain spectrum along a short optical fibre, obtained using a $35 \mathrm{~cm}$ spatial resolution and a $16 \mathrm{X}$ time trace averaging.

Right: Gain spectrum obtained at one position using the experimental conditions, showing the excellent measurement quality and the low noise obtained using the novel configuration. 


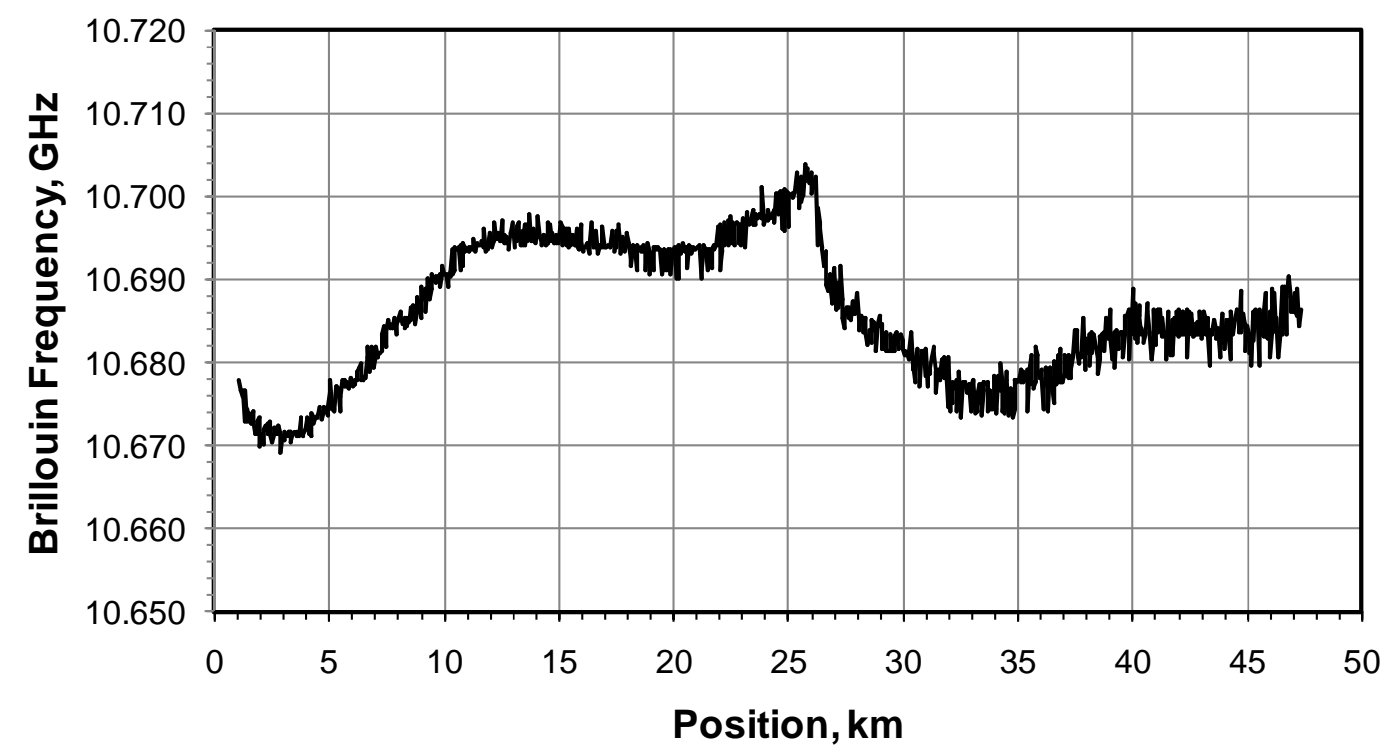

Fig. 3. Measured distribution of the Brillouin frequency along a $47 \mathrm{~km}$ dispersion-shifted optical fibre, obtained using a $3.5 \mathrm{~m}$ spatial resolution and a 256X time trace averaging. The fluctuations of the Brillouin frequency result from fibre inhomogenity and a varying winding tension of the fibre. A $1 \mathrm{MHz}$ resolution on the Brillouin frequency is obtained on the first $30 \mathrm{~km}$ and a $2 \mathrm{MHz}$ resolution is secured over the full range.

\section{CONCLUSIONS}

We have developed a novel BOTDA configuration able to perform a distributed measurement of the Brillouin gain spectrum using a single laser source. This configuration is designed to minimize all sources of intensity noise, resulting in extreme performances in terms of range, spatial resolution and acquisition time. We have obtained a spectral resolution of $3.5 \mathrm{~m}$ over a $47 \mathrm{~km}$ fibre length. It must be pointed out that the range is actually limited by the modulation instability that actively spreads the spectrum of the pump pulse and longer fibre lengths can certainly be measured using a fibre showing a normal group velocity dispersion. A new passive polarization scrambler made of an unbalanced MachZehnder interferometer was implemented and made possible the measurement of Brillouin frequencies with a maximum variation of $33 \mathrm{kHz}$ and much reduced Brillouin gain oscillations.

\section{ACKNOWLEDGMENTS}

Financial support from the European COST Action 299 FIDES, Spanish Comisión Interministerial de Ciencia y Tecnología within project TEC2004-05936-C02 and FEDER funds is acknowledged.

\section{REFERENCES}

1. T.Horigushi, M.Tateda, "Optical- fiber-attenuation investigation using stimulated Brillouin scattering between a pulse and a continuous wave", Optics Letters, 14, p.408, 1989.

2. X. Bao, J. Dhliwayo, N. Heron, D.J. Webb, D.A. Jackson, "Experimental and theoretical studies on a distributed temperature sensor bases on Brillouin scattering", J. Lightwave Technol., 13, p. 1340, 1995.

3. L. Thévenaz, S. Le Floch, D. Alasia and J. Troger, "Novel schemes for optical signal generation using laser injection locking with application to Brillouin sensing," Measurement Science and Technology 15, 1519-1524 (2004).

4. M. Niklès, L. Thévenaz and P. Robert, "Brillouin Gain Spectrum Characterisation in single-mode optical fibres," Journal of Lightwave Technology, 15(10) 1842-1851 (199 ).

5. M. Niklès, L. Thévenaz, P. Robert, "Measurement of the distributed Brillouin-gain spectrum in optical fibres by using a single laser source," Technical Digest, OFC'94, San Jose, CA, paper WF1, 89-90 (1994).

6. M. Niklès, L. Thévenaz and P. Robert, "Simple distributed fibre sensor based on Brillouin gain spectrum analysis," Optics Letters, 21(10) 万8-760 (1996). 